\title{
Diffractive Dijets in DIS and PHP
}

\author{
Matthias Mozer ${ }^{1}$ \\ 1- Vreije Universiteit Brussel - IIHE \\ Pleinlaan 2, 1050 Brussel - Belgium
}

\begin{abstract}
I present measurements of dijet cross-sections in diffractive DIS and photoproduction taken with the $\mathrm{H} 1$ detector at the HERA accelerator. Diffractive events were identified by a rapidity gap selection. The resulting differential cross sections are compared to QCD calculations in NLO, based on parton densities extracted from inclusive diffraction. Additionally a fit of diffractive parton densities to the combined data sets of the inclusive $F_{2}^{D}$ measurement and the dijet data was performed. This leads to reduced uncertainties for the gluon density.
\end{abstract}

\section{Introduction}

Theoretically it is expected that the cross sections of diffractive deep-inelastic scattering (DIS) factorises into universal diffractive parton distributions and process dependent hard scattering coefficients [2]. Diffractive parton densities have been determined from DGLAP QCD fits to inclusive diffractive HERA data $[3,4]$ and have been found to be dominated by the gluon distribution. Diffractive dijet production is directly sensitive to the gluon component of the diffractive exchange and has been shown - for DIS [5] - to be in decent agreement with the QCD fits to the inclusive diffractive data. In this paper, a new measurement of diffractive dijet cross sections in deep inelastic scattering is presented, based on data collected with the H1 detector at HERA. A combined NLO QCD fit is performed to the differential dijet cross sections and the inclusive diffractive structure function $F_{2}^{D}$ in order to determine the diffractive quark and gluon distributions with higher accuracy.

However, applying this approach in LO QCD calculations to predict diffractive cross sections for dijet production in $p \bar{p}$ collisions at the Tevatron leads to an overestimation of the observed rate by approximately one order of magnitude [6]. This discrepancy has been attributed to the presence of the additional beam hadron remnant in $p \bar{p}$ collisions, which leads to secondary interactions and a breakdown of factorisation. The suppression, often characterised by a 'rapidity gap survival probability', cannot be calculated perturbatively and has been parameterised in various ways (see for example [7]).

The transition from deep-inelastic scattering to hadron-hadron scattering can be studied at HERA in a comparison of scattering processes in DIS and in photoproduction. Processes in which a real photon participates directly in the hard scattering are expected to be similar to the deep-inelastic scattering of highly virtual photons. By contrast, processes in which the photon is first resolved into partons which then initiate the hard scattering resemble hadron-hadron scattering. In this article, the final results of diffractive dijet cross sections in DIS and photoproduction are presented, based on data collected with the H1 detector at HERA.

\section{Experimental Procedure}

The detector setup and selection criteria for the comparison of dijet cross sections in DIS and photoproduction are described in detail in [5]. The cross scetion measurement is measured 
similarly, the most notabe differences being a wider y-range (0.1-0.7) and a tighter cut on the transverse momentum of the hardest jet $(5.5 \mathrm{GeV})$.

\section{Rapidity Gap Survival}

While diffractive dijet production in DIS shows reasonable agreement with NLO QCD calculations based on the factorisation approach, which is not the case in photoproduction. This disagreement is often interpreted as a 'rapidity gap survival probability' smaller than one due to secondary interactions of spectator partons and measured from the difference between the measured cross section and perturbative NLO QCD calculations. With this method, the uncertainty of the diffractive parton densities used for the prediction limits the accuracy of the measurement. Figure 1 shows the double ratio $\frac{\left((d \sigma / d W)_{d a t a}\right) /\left((d \sigma / d W)_{N L O}\right)^{P H P}}{\left((d \sigma / d W)_{d a t a}\right) /\left((d \sigma / d W)_{N L O}\right)^{D I S}}$ in which the parton density uncertainties mostly cancel. This shows that diffractive dijet production in photoproduction is significantly suppressed by a factor $\sim 0.5$ compared to perturbative calculations. Most surprisingly, the suppression shows no kinematic dependence.

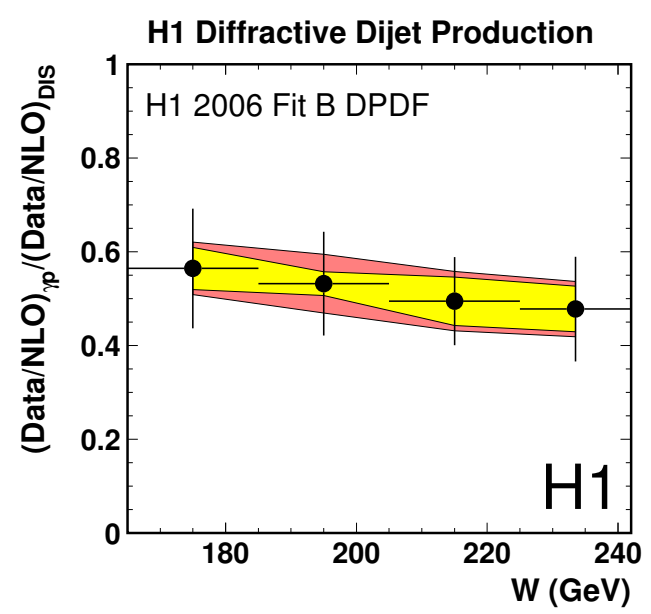

Figure 1: The double ratio $\frac{\left((d \sigma / d W)_{d a t a}\right) /\left((d \sigma / d W)_{N L O}\right)^{P H P}}{\left((d \sigma / d W)_{d a t a}\right) /\left((d \sigma / d W)_{N L O}\right)^{D I S}}$. The error bars on the points represent the statistical uncertainty, while the inner error band shows the experimental systematic uncertainties and the outer band represents the uncertainties connected to the NLO calculation.

\section{Parton Density Fit}

In DIS the differential dijet cross section in $z_{\mathbb{P}}$ is used in the fit in 4 bins of the scale variable $p_{\perp}^{\star 2}+Q^{2}$ to constrain the gluon density, where $p_{\perp}^{\star}$ is the transverse momentum of the hardest jet. These measured cross sections are shown in Figure 2 (left). Additionally the inclusive data sample of a previous $\mathrm{H} 1$ analysis [4] is used to constrain the quark density and the gluon density a low momentum fraction. A part of the $F_{2}^{D}$ measurements is shown in Figure 2 (right) together with the final NLO prediction. 

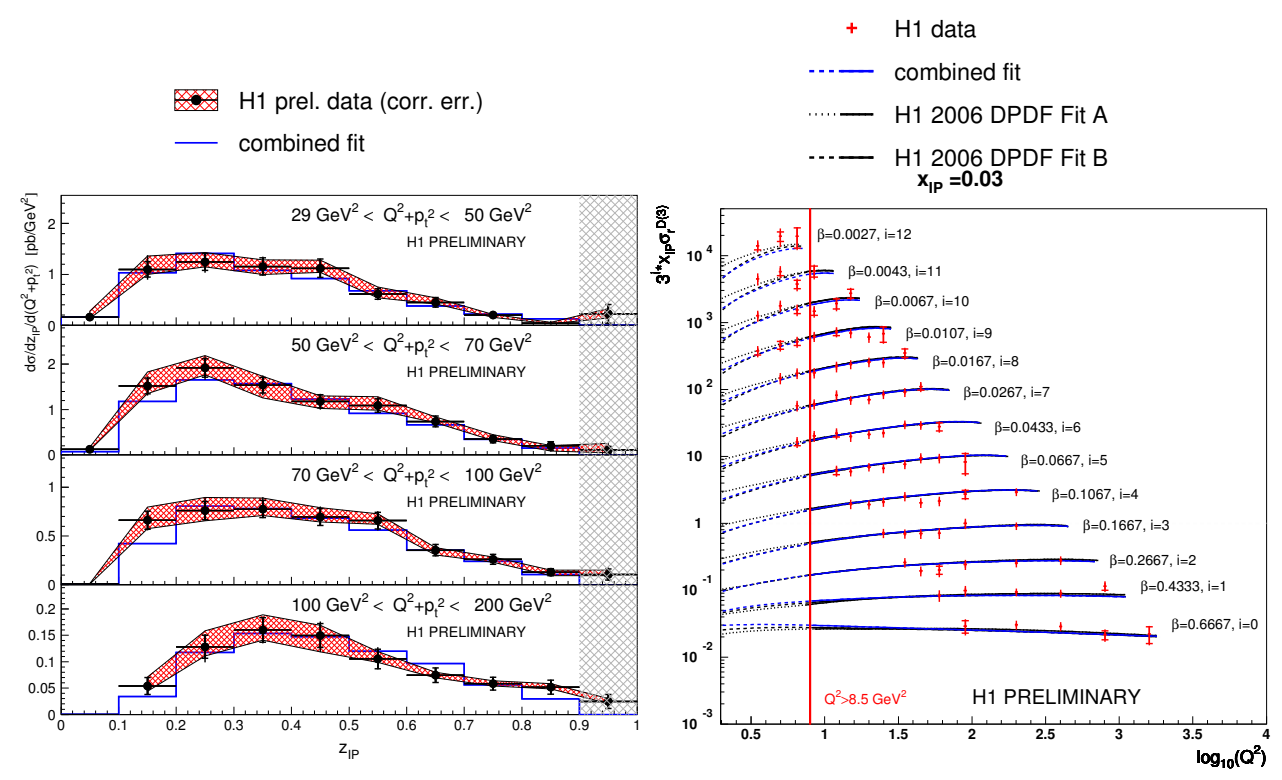

Figure 2: left: Cross section of diffractive dijets doubly differential in in $z_{\mathbb{P}}$ and the scale $\mu=Q^{2}+p_{\perp}^{\star 2}$. The data are shown as black points with the inner and outer error-bar denoting the statistical and uncorrelated systematic uncertainties respectively. The red hatched band indicates the correlated systematic uncertainty. The blue line shows the NLO QCD prediction based on the combined fit. right: The $\beta$ and $Q^{2}$ dependence of the diffractive reduced cross section $\sigma_{r}^{D(3)}$ multiplied by the pomeron momentum fraction $x_{\mathbb{P}}$ at $x_{\mathbb{P}}=0.03$. The inner and outer error-bars on the data points represent the statistical and total uncertainties, respectively. The data are compared to the results of the combined fit for $E_{p}=820 \mathrm{GeV}$, which is shown as blue lines. The dashed line indicates the prediction in kinematic regions that did not enter into the fit. The two black lines indicate the predictions of the H1 2006 DPDF fit.

The parton densities are parameterised as of momentum fraction $z$ at a starting scale $Q_{0}^{2}$ as $A \cdot z^{B} \cdot(1-z)^{C}$ and evolved to higher scales by the DGLAP equations in NLO. Here, $A, B$ and $C$ are free parameters, determined in the fit. Additionally the Regge intercept $\alpha(0)$ of the pomeron flux factor and the normalisation of the sub-leading reggeon exchange enter the fit as free parameters. From these parton densities the reduced cross section for inclusive diffractive DIS is computed in NLO as well as the dijet cross section (using the nlojet++ program).

The fit has a high quality as shown by the overall value $\chi^{2} / d f=0.89$ which splits into $\chi^{2} / d f=27 / 36$ for the dijet cross sections and $\chi^{2} / d f=169 / 190$ for $F_{2}^{D}$. The resulting parton distributions are shown in Figure 3.

As the NLO QCD DGLAP evolution is able to describe both the shape and scaling violations of $F_{2}^{D}$ and the dijet cross sections consistently, we conclude that QCD factorisation in DIS is valid in our kinematic region. The data has allowed for the first time to determine 
both the diffractive gluon and the singlet quark distribution with good accuracy in the range $0.1<z_{\mathbb{P}}<0.9$.
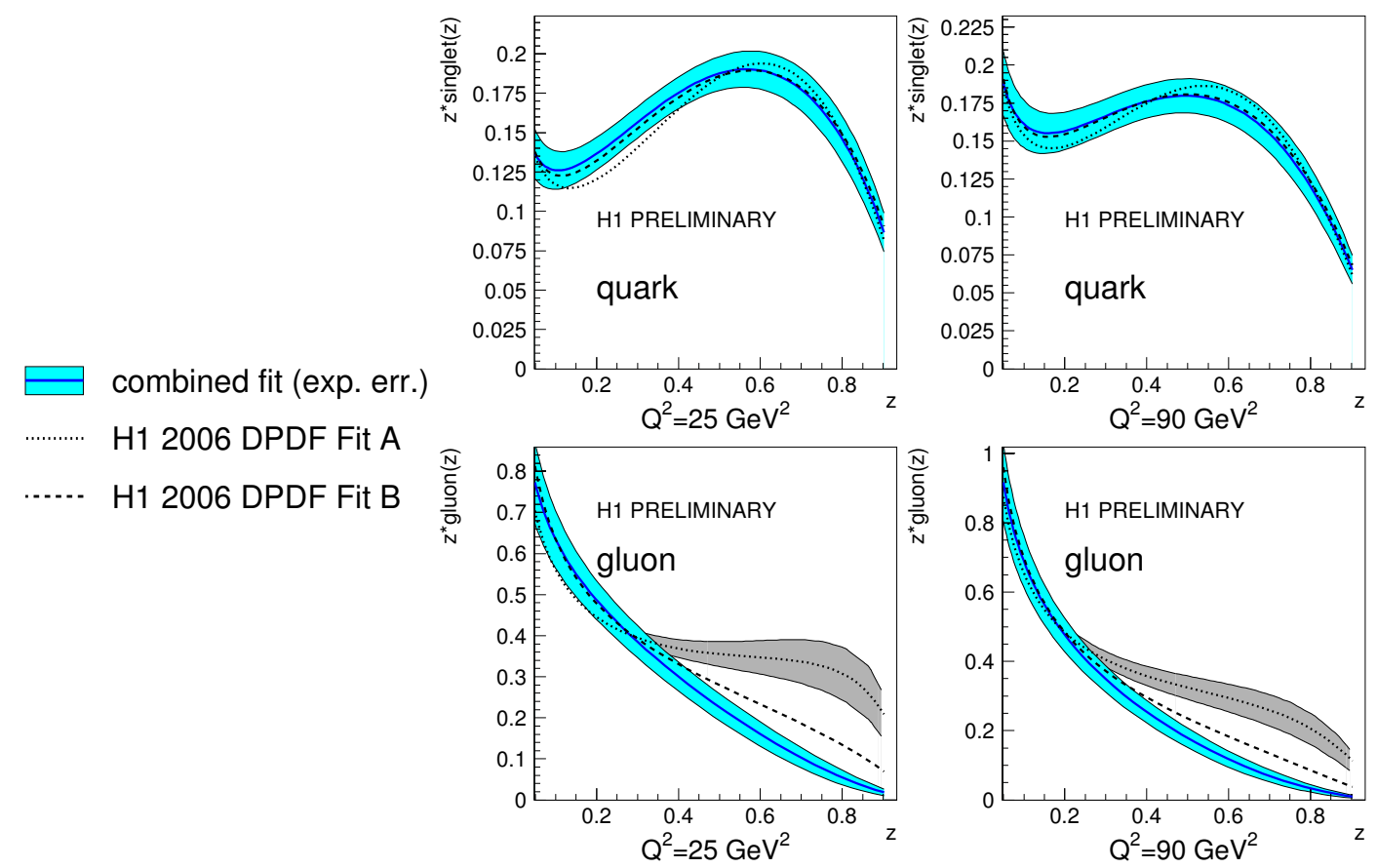

Figure 3: The diffractive singlet density (top) and diffractive gluon density (bottom) for two values of the hard scale $\mu$ : $25 \mathrm{GeV}^{2}$ (left) and $90 \mathrm{GeV}^{2}$ (right). The blue line indicates the combined fit, surrounded by the experimental uncertainty band in light blue. The two dashed lines show the two fit results from [4] for comparison.

\section{References}

[1] Slides:

http://indico. cern. ch/contributionDisplay $\cdot$ py? contribId=62\&sessionId=7\&conf Id=9499

[2] J. Collins, Phys. Rev. D57 (1998) 3051 and erratum-ibid. D61 (2000) 019902.

[3] C. Adloff et al. [H1 Collaboration], Z. Phys. 76 (1997) 613

[4] A. Aktas et al. [H1 Collaboration], Eur. Phys. J. C 48 (2006) 715 [hep-ex/0606004].

[5] A. Aktas et al. [H1 Collaboration], submitted to Eur. Phys. J. C [hep-ex/0703022].

[6] CDF Collaboration, T. Affolder et al., Phys. Rev. Lett. 84 (2000) 5043.

[7] A. Kaidalov, V. Khoze, A. Martin, M. Ryskin, Phys. Lett. B567 (2003) 61 